\title{
Heritable influences on the mind and brain
}

\author{
In this issue, we highlight examples of the growing capability of genetic epidemiology and its intersection with \\ genomic data to identify the underpinnings of the functions, predispositions and vulnerabilities of the human brain. \\ In particular, we are publishing three studies into intelligence, neuroticism and epilepsy with the potential to guide \\ interventions in education, neuroscience and medicine, respectively.
}

\author{
$\mathrm{n}$ his superb book on heredity, She \\ Has Her Mother's Laugh, Carl Zimmer \\ discusses how the proper understanding \\ of heritable influences on intelligence and \\ the related trait of educational attainment \\ could be used to guide the design and \\ evaluation of improved educational \\ strategies. Intelligence, like height, is highly \\ heritable, polygenically inherited and \\ depends environmentally upon nutrition \\ and opportunity. For this new knowledge \\ to be used effectively, he argues, the very \\ real genetic influences should be considered \\ constitutional potential, rather than the \\ more frequently grasped concept of \\ genetic essence. \\ In a Letter in this issue, Jeanne Savage, \\ Philip Jansen and colleagues identify \\ genetic variants accounting for about $5 \%$ \\ of the variance in intelligence tested in the \\ populations they studied, amounting to 205 \\ associated loci, and implicate 1,016 genes \\ by their associated position, expression \\ quantitative traits and regulatory enhancer- \\ promoter contacts. The constituents \\ of fluid (test-taking) intelligence such \\ as problem solving, working memory, \\ time management and processing speed, \\ attending to and manipulating new \\ information in the environment, and \\ reasoning are all highly correlated. The \\ genetic differences underlying the trait's \\ distribution in the population appear in \\ this study to be distinctive from the set of \\ genes that underlie intellectual disability
}

and developmental delay. The set of genes contributing to intelligence are strongly implicated to function in subsets of brain cells and the developmental processes that build the human brain.

Neuroticism is a personality trait that combines aspects of depression and worry, and which predisposes to diagnoses of mental illnesses such as anxiety, depression and schizophrenia. In a second Letter, Mats Nagel, Philip Jansen and colleagues identify 136 loci implicating 599 genes involved in several brain regions, sorting the genetic basis for the trait into two main subclasses via the variants that contribute to the subtraits of depressed affect and worry. They also identify subtypes of brain cells contributing to the personality trait (serotonergic and dopaminergic neurons) that are therapeutic targets in mood disorders and mental illnesses. As with intelligence, the correlation of these behavior traits with health outcomes will enable better understanding of predisposition to health and disease, as well as provide a platform upon which to evaluate interventions. The research implications of these findings should be important in enabling new avenues of neuroscience research. For example, the polygenic scores associated with the highest and lowest levels of these traits might be used to compare genetically similar and contrasting groups of individuals performing cognitive tasks or in different mood states, using functional imaging and electrophysiological measurements to examine the activities of neural systems, circuits and pathways.

In an Analysis also published in this issue, Henrike Heyne and colleagues found 33 genes with an excess of de novo mutations among 1,942 individuals ( $29 \%$ of 6,753 family trios) with neurodevelopmental disorders who also had epilepsy. Two of these genes had not previously been found mutated in epilepsy. The similarity of the genetic spectrum of unspecific epilepsy in neurodevelopmental disorders to that of epileptic encephalopathy suggests that an up-to-date sequencing panel of all genes recurrently mutated in epilepsy is warranted in order to identify individuals with neurodevelopmental disorders who would benefit from antiepileptic treatment to prevent encephalopathy. This large, systematic survey was useful in recommending standardization of the set of genes to be used in clinical testing, not only in including newly identified genes for this panel, but also in deprioritizing a large number of genes present on some panels for which there is insufficient evidence of pathogenicity in epilepsy. So, this study found new biology and informs more precise genomic medicine.

Published online: 28 June 2018 https://doi.org/10.1038/s41588-018-0172-2 